\title{
Consciência ambiental e percepção sobre os resíduos sólidos pelos residentes da Cidade de Coremas, Paraíba
}

\section{Environmental Awareness and perception about solid waste by the residents of the City of Coremas, Paraíba}

\author{
Carlos Sergio Araújo dos Santos ${ }^{1 *}$, Daniel Jackson Andrade de Sousa ${ }^{2}$, Gabriel Carlos Moura Pessôa ${ }^{3}$, Ricardo Ricelli \\ Pereira de Almeida ${ }^{4}$, Alan Dél Carlos Gomes Chaves ${ }^{5}$
}

\begin{abstract}
Resumo: Resíduos sólidos são todos os restos sólidos ou semi-sólidos das atividades humanas ou não-humanas, que embora possam não apresentar utilidade para a atividade fim de onde foram gerados, podem virar insumos para outras utilizações. Objetivou-se avaliar o comportamento da população à respeito das atitudes e percepção com relação aos resíduos sólidos na cidade de Coremas, Paraíba. Os dados referentes a atitudes e percepção dos atores sociais foram coletados na cidade de Coremas por meio de questionários, previamente estruturados com perguntas de múltipla escolha a fim de conhecer o perfil dos entrevistados através das seguintes variáveis: sexo, faixa etária, escolaridade, estado civil e renda. Foi realizada uma análise descritiva para verificar a percepção dos residentes no município segundo as variáveis relacionadas aos resíduos sólidos. A relação entre variáveis socioeconômicas e o comportamento ambiental referente aos resíduos sólidos dos moradores da cidade de Coremas foi verificada por meio do teste qui-quadrado de independência, mostrando que houve forte dependência entre essas variáveis, sugerindo que, a escolaridade, a renda e a localização dos residentes influenciaram em suas atitudes e percepções ambientais naquele município.
\end{abstract}

Palavras-chave: Percepção ambiental; Meio ambiente; Qualidade de vida; Lixo.

Abstract: Solid wastes are all the solid or semi-solid residues of human or non-human activities that, although do not show utility for the order in which they were generated, can become to inputs for other activities. The study's aim was to evaluate the population's behavior about the attitudes and the perception connected to the solid wastes in the city of Coremas, Paraíba state. The data on attitudes and perceptions of the social actors were collected in the city of Coremas through questionnaires, previously structured with multiple choice questions in order to know the profile of the interviewed using the following variables: gender, age, education, marital status and income. The Descriptive analysis were performed to verify the perception of residents in the city according to the related variables to solid wastes. The relationship between socioeconomics variables and environmental performance related to solids wastes from residents of Coremas were verified by the chi-square test of independence, showing that there was a strong dependency between these variables, suggesting that the educational level, the income and the location of residents influenced in their attitudes and environmental perceptions in that city.

Key words: Environmental awareness; Environment; Quality of life; Trash.

\footnotetext{
*Autor para correspondência

Recebido para publicação em 13/09/2016; aprovado em 02/01/2017

${ }^{1}$ Professor de Estatística, Universidade Federal de Campina Grande, Pombal; Fone, E-mail: carlos.santos@ccta.ufcg.edu.br.

${ }^{2}$ Graduando em Engenharia Ambiental, Universidade Federal de Campina Grande, E-mail: danielcoremas@gmail.com

${ }^{3}$ Graduando em Engenharia Ambiental, Universidade Federal de Campina Grande, E-mail: pessoagabriel553@gmail.com

${ }^{4}$ Mestrando em Sistemas Agroindustriais, Universidade Federal de Campina Grande, E-mail: ricelli2008@bol.com.br

${ }^{5}$ Doutorando de Engenharia Agrícola, Universidade Federal do Recôncavo Baiano, E-mail: alan.delcarlos@hotmail.com
} 


\section{INTRODUÇÃO}

Os impactos causados pela falta de gerenciamento dos resíduos sólidos são de grande proporção na sociedade, segundo Left (2001), a compreensão de ambiente deve pautar na racionalidade ambiental, na transdisciplinaridade, pensando não como um sinônimo de natureza, e sim como base de interações entre o meio físico e biológico com as sociedades e a cultura produzida por esses membros. Os impactos são ocasionados desde o momento de interação do homem com o meio ambiente, isso implica num impacto que pode ocasionar um dos maiores problemas hoje na sociedade, que é a disposição inadequada dos resíduos sólidos.

$\mathrm{O}$ atual padrão de consumo da sociedade, atrelado aos efeitos das propagandas de marketing empresarial, influencia a comprar cada vez mais. Frequentemente, utensílios e materiais que ainda poderiam ser reciclados são tratados como resíduo, devido a hábitos culturais ou falta de conhecimento sobre impactos ambientais decorrentes (CARDOSO FILHO, 2012).

As cidades brasileiras apresentam-se com um padrão deficiente quando o tema pesquisado é saneamento ambiental, principalmente nas cidades da região Nordeste. Pode-se destacar então o gerenciamento dos resíduos sólidos, sabe-se que a grande maioria de resíduos produzidos pela população das cidades não passam por nenhum processo de separação para receber uma disposição final. Acabando então em lixões, dificultando o seu aproveitamento econômico e geração de renda (IBGE, 2015).

Segundo a lei 12305- 2010 Política Nacional dos Resíduos Sólidos: Resíduos Sólidos são definidos como "Materiais, substâncias, objeto ou bem descartado resultante de atividades humanas em sociedade, a cuja destinação final se procede, se propõe proceder ou se está obrigado a proceder, nos estados sólido ou semissólido, bem como gases contidos em recipientes e líquidos cujas particularidades tornem inviável o seu lançamento na rede pública de esgotos ou em corpos d'água, ou exijam para isso soluções técnicas ou economicamente inviáveis em face da melhor tecnologia disponível" (BRASIL, 2010).

Diante de inúmeras conferências para discutir melhorias para o meio ambiente, obter hoje, a redução na quantidade de resíduos sólidos produzidos diariamente chega a ser um grande desafio. Segundo Mucelin e Bellini (2008), o consumo frequente de produtos industrializados está relacionado à constante produção de lixo. O lixo produzido nas grandes cidades ocorre de forma intensa não sendo impossível assimilar uma cidade sem considerar a problemática gerada até a disposição final dos resíduos sólidos.

$\mathrm{O}$ manejo inadequado dos recursos naturais se torna um fator de grande preocupação, atrelado a isso, encontra-se o mau gerenciamento dos resíduos sólidos. A falta desse gerenciamento e o desgaste contínuo dos recursos naturais vão contra todo o objetivo da Política Nacional do Meio Ambiente expressa na Lei 6.938/81 (BRASIL, 1981). Segundo Canter (1977), o impacto ambiental é definido como qualquer alteração no sistema ambiental físico, químico, biológico, cultural e socioeconômico que seja conferido por atividades antrópicas, relativas às alternativas em estudo para satisfazer as necessidades de um projeto.

Diante dessa problemática, a educação ambiental vem sendo bastante citada como ferramenta crucial na mudança dos costumes do brasileiro com a disposição final dos resíduos sólidos. Assim, faz-se necessário formar cidadãos conscientes quanto a preservação do meio ambiente. Aumentando, assim sua percepção para o gerenciamento dos resíduos sólidos. Desta forma, objetivou-se diagnosticar o comportamento da população da cidade de Coremas, Paraíba, por meio das suas atitudes e percepções em relação aos resíduos sólidos.

\section{MATERIAL E MÉTODOS}

O presente estudo foi realizado no município de Coremas no estado da Paraíba, localizado na microrregião de Piancó. (Latitude $07^{\circ} 00^{\prime} 50^{\prime}$ ' Sul, Longitude $37^{\circ} 56^{\prime} 45^{\prime}$, Oeste). O município está incluído na área geográfica de abrangência do Semiárido brasileiro, definida pelo Ministério da Integração Nacional em 2005.

Para a verificação do diagnóstico da percepção dos moradores da cidade quanto aos resíduos sólidos, foi realizada uma pesquisa qualitativa estruturada com perguntas categorizadas e abertas, buscando conhecer o perfil dos entrevistados através de variáveis como sexo, faixa etária, escolaridade, estado civil e renda, e a percepção e conhecimento ambiental com questões sobre limpeza pública e meio ambiente.

A amostragem contemplou 577 domicílios, de acordo com o público alvo, totalizando 577 autores sociais (um indivíduo por moradia) com nível de confiança de $95 \%$, e, erro de $4 \%$. Os dados referentes à percepção ambiental dos atores sociais foram coletados por meio de questionários, previamente estruturados com perguntas de múltipla escolha a fim de conhecer o perfil dos entrevistados através das seguintes variáveis: sexo, faixa etária, escolaridade, estado civil e renda. No que tange à pesquisa de campo, foi aplicado o questionário "O saneamento básico na cidade de Coremas (PB)".

Para traçar o perfil dos entrevistados foram obtidas estatísticas descritivas, com número e percentagem para cada variável estudada a fim de avaliar a distribuição das respostas.

Para que o estabelecimento de propostas de educação ambiental em uma área impactada seja eficiente, é importante detectar os níveis de percepção ambiental da população da referida área para que se conheçam seus valores atitudes, condutas e como os impactos sofridos influenciaram na percepção desses indivíduos. Desta foram, foi realizado o teste Qui-quadrado de independência considerando um nível de significância de 5\% para verificar a relação das variáveis escolaridade, renda e bairro com a variável atitude do pesquisado a respeito do destino do lixo gerado. Também foi realizado um teste de qui-quadrado de independência para averiguar se há relação entre o destino do lixo coletado e se o entrevistado possui coleta.

O teste Qui-quadrado de independência é utilizado para testar a hipótese de que duas variáveis não estão relacionadas, isto é, são independentes. A hipótese nula afirma que as variáveis em questão são independentes (não há relação entre elas), a hipótese alternativa afirma que as variáveis não são independentes (há relação entre as variáveis). 


\section{RESULTADOS E DISCUSSÃO}

Observou-se que a amostra apresentou diversos níveis de escolaridade que contemplavam o ensino fundamental e médio, sem escolaridade e o nível superior num universo de 577 entrevistados. A pesquisa revelou que a maioria dos entrevistados possuía um nível de escolaridade muito baixo, o número de pessoas que concluíram ou estão em processo de conclusão do ensino fundamental foi de $55 \%$, o ensino médio foi de $17 \%$, enquanto as pessoas que tem ensino superior foi de $9 \%$, o percentual de analfabetos amostrados na pesquisa de $1 \%$. O número de analfabetos no município é considerado elevado e a grande maioria dos entrevistados possui baixo nível de escolaridade.

De acordo com os resultados, foi possível observar o perfil dos entrevistados, apresentando uma predominância de mulheres com percentual $72 \%$ da amostra estimada, enquanto que $28 \%$ de homens. Na pesquisa também foi indagado sobre o estado civil dos entrevistados mostrando que $66 \%$ das pessoas encontram-se casadas, enquanto $34 \%$ solteiras. Verificou-se também uma faixa etária de 20 a 30 anos correspondendo a um percentual $(30,1 \%)$, outra parcela com idade entre 36 e 45 anos o equivalente a $(34,7 \%)$ e com 46 ou mais anos correspondendo um percentual de $(35,2 \%)$.

Observa-se na Figura 1 referente a coleta de resíduos na cidade, que grande parte das pessoas relataram possui coleta de resíduos sólidos em seu domicílio, representando um percentual de $83 \%$ dos entrevistados. O percentual que afirmou não ter coleta dos resíduos está ligado a bairros de regiões da cidade que são afastados do perímetro urbano, onde os autores sociais da pesquisa afirmaram que queimam o lixo produzido, essa prática pôde ser observada na Figura 2 onde esse percentual correspondeu a 9,7\% dos entrevistados, sendo proveniente de uma deficiência do recolhimento de lixo por parte do órgão responsável pelo saneamento básico da cidade. Ainda é possível verificar que grande parte dos residentes separa o lixo produzido para a coleta, representando 88,2\% dos entrevistados, assim, tem-se uma consciência ambiental plausível no que tange a atitude dos moradores relacionada ao destino dos resíduos produzidos de forma a não agredir o meio ambiente (Figura 2).

Figura 1. Percentual dos entrevistados que possuem coleta de resíduos sólidos em seu domicílio na cidade de Coremas, Paraíba.

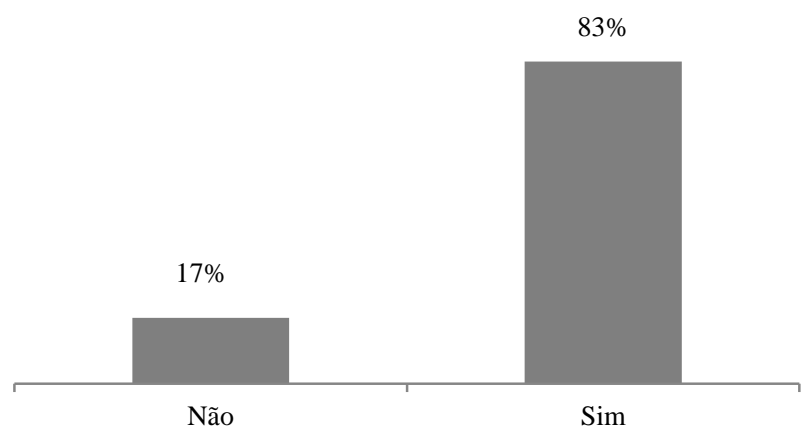

Figura 2. Atitude dos entrevistados a respeito do lixo produzido em seu domicílio na cidade de Coremas, Paraíba.

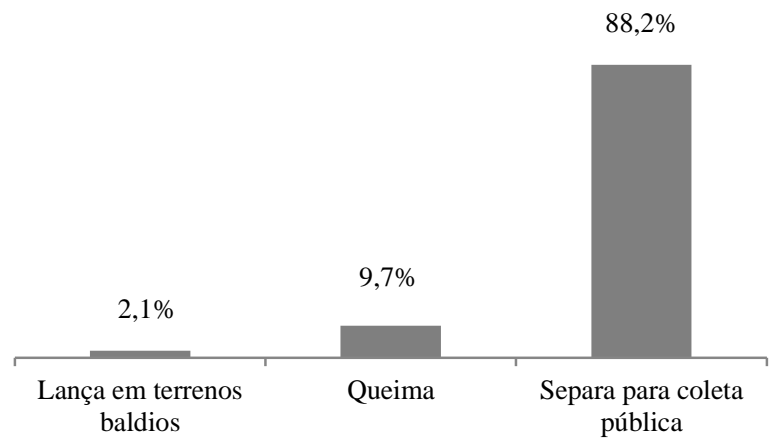

$\mathrm{Na}$ Figura 3 verifica-se a atitude dos entrevistados quanto ao destino dos resíduos produzidos na rua, onde se pode observar que há uma consciência do destino do resíduo, em que 76,6\% dos entrevistados jogam os resíduos no lixo, já a atitude de guardar no bolso 15,6 c das pessoas entrevistadas afirmaram que realizam essa atitude, e por fim $7,8 \%$ jogam no chão, esses percentuais mostram um desenvolvimento da consciência ambiental e sanitária das pessoas, sinalizando que a coleta e o lançamento do resíduo produzido é jogado no lixo o que caracteriza como uma verdadeira conscientização para com o destino do mesmo portanto, há uma preocupação por parte da população com os princípios fundamentais do saneamento ambiental.

Figura 3. Atitude dos entrevistados a respeito do lixo produzido nas ruas na cidade de Coremas, Paraíba.

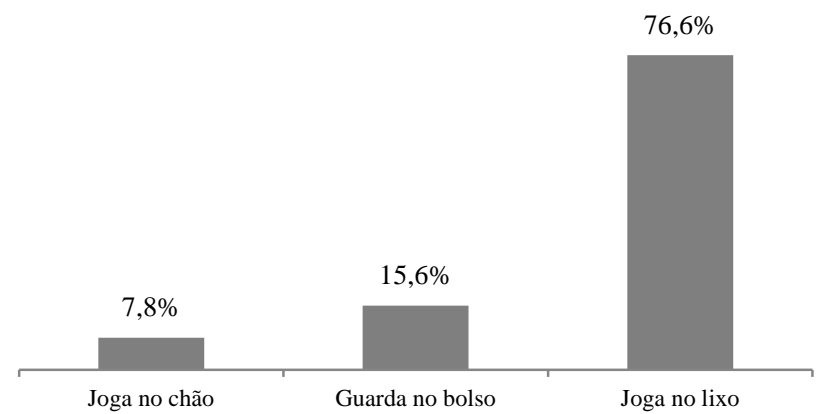

Para efeito de verificação da relação entre a atitude do pesquisado com o destino do lixo gerado com as variáveis escolaridade e renda, foi aplicado o teste de qui-quadrado de independência ao nível de 5\% de significância. Mediante o teste foi possível identificar que as variáveis são dependentes (Tabela 1). Assim percebe-se que as pessoas cujo apresentam uma maior renda contribuem mais para a preservação do meio ambiente, sendo essas classes mais providas de educação e conscientização ambiental, quando se tem uma população que contribui com a limpeza urbana as políticas internas da cidade se tornam mais eficazes.

Tabela 1. Estatísticas do teste qui-quadrado para verificar relação entre as variáveis Renda e Destinação do lixo gerado em seu domicílio.

\begin{tabular}{lr}
\hline Teste Qui-Quadrado & Valor \\
\hline Estatistica X & $32,82233908^{*}$ \\
Graus de Liberdade & 4 \\
P-Valor & $1,29882 \mathrm{E}-06$ \\
\hline *ejeitar $\mathrm{H}_{0}$ (Hipótese nula) se $\mathrm{X}^{2}>9,488$ &
\end{tabular}


Segundo Bidone e Povinelli (1999) a média da geração per capita de resíduos sólidos é função da quantidade de resíduos coletados em uma cidade dividida pela população beneficiada por esses serviços. Ela se altera em função de fatores culturais, hábito de consumo, padrão de vida e a renda familiar que define o poder de compra.

Franco (2000) afirma que a implantação de uma coleta seletiva deve começar pelos bairros que apresentem uma melhor infraestrutura, ou seja, os bairros onde se concentram uma população com maior poder aquisitivo, devido a presença de todos os serviços públicos básico como, água, luz, telefone, coleta de lixo, estrutura de esgoto e vias pluviais.

Um projeto de coleta de resíduos pela suas especificidades, apresenta no seu desenvolvimento uma série de dificuldades. Não depende apenas de inciativas de órgãos gerenciadores da limpeza urbana e da ação de alguns de seus técnicos, pois exige para seu sucesso a participação ativa da população. No caso de Coremas, a necessidade da participação de baixa renda na contribuição do trabalho de limpeza urbano, assim como foi mencionado, o município deverá investir nas políticas de públicas de educação e informativos a fim de melhorar a situação nos bairros menos providos de recurso.

A dependência entre as variáveis escolaridade e atitude do entrevistado quanto à disposição dos resíduos também se confirmou quando aplicou-se o teste qui-quadrado, onde pode ser observado que quanto maior o nível de escolaridade (analfabeto > fundamental > médio > superior) maior foi a colaboração em não jogar lixo na rua, guardando no bolso e depositando no cestos de lixo, sendo encontrada em maior proporção na classe com maior nível de escolaridade, com isso torna-se necessário a implantação de políticas de educação para as classes menos favorecidas.

A sociedade quando educada e informada exerce papel fundamental diante de problemas de ordem social e ambiental, despertando para a realidade, e responsabilidade de cada individuo, neste contexto a educação ambiental tornase ferramenta de grande importância pelas várias nuances a possibilidades de trabalhar tanto com crianças, jovens ou adultos.

Tabela 2. Estatísticas do teste qui-quadrado para verificar relação entre as variáveis Escolaridade e Destinação do lixo gerado na rua.

\begin{tabular}{lr}
\hline Teste Qui-Quadrado & Valor \\
\hline Estatistica X & $53,76269048^{*}$ \\
Graus de Liberdade & 6 \\
P-Valor & $8,23637 \mathrm{E}-10$ \\
\hline *Rejeitar $\mathrm{H}_{0}$ (Hipótese nula) se $\mathrm{X}^{2}>12,592$ &
\end{tabular}

As práticas de Educação Ambiental aliada à coleta seletiva do lixo são iniciativas que representam grande importância do ponto de vista ambiental e social minimizando os problemas na cidade. $\mathrm{O}$ comportamento dos cidadãos em relação ao meio ambiente é intrínseco do exercício da cidadania para a construção e um ambiente saudável e equilibrado, buscando o bem comum da sociedade.

Cornieri e Fracalanza (2010) afirmam que as atividades de educação ambiental relacionadas aos resíduos sólidos devem ser melhoradas nos municípios, ampliando-se o campo de transmissão dessas informações, afim de que a maioria da população saiba um pouco sobre a gestão de resíduos sólidos no seu município e assim possa estar sempre contribuindo para a mesma.

Jacobi (2005) afirma que é preciso desenvolver meios que permitem acesso as informações, por meio de uma ação mais eficaz dos gestores públicos como forma de minimizar os danos ambientais.

Com a finalidade de investigar a associação entre as variáveis lixo coletado com o bairro onde reside e a variável se possui coleta de resíduos, também foi aplicado o teste de qui-quadrado de independência ao nível de $5 \%$ de significância.

Através do teste verificou-se que as variáveis bairro versus possui coleta são dependentes (Tabela 3). Assim, é possível confirmar a relação de quanto mais carente é o bairro, menor poder aquisitivo e assim mais propicio a deficiência na coleta de lixo. Esta falta de serviço em alguns bairros da cidade de Coremas (PB) mostra o não cumprimento da Lei Federal $n^{\circ} 11.445 / 07$, chamada de Lei Nacional do Saneamento Básico que estabelece a universalização do acesso a todos os serviços contidos no contexto de saneamento básico, onde se enquadra a coleta de resíduos sólidos (BRASIL, 2007).

Tabela 3. Estatísticas do teste qui-quadrado para verificar relação entre as variáveis Bairro e Destinação do lixo gerado em seu domicílio.

\begin{tabular}{lr}
\hline Teste Qui-Quadrado & Valor \\
\hline Estatistica X & $48,35854075^{*}$ \\
Graus de Liberdade & 5 \\
P-Valor & $3,00095 \mathrm{E}-09$ \\
\hline
\end{tabular}

*Rejeitar $\mathrm{H}_{0}$ (Hipótese nula) se $\mathrm{X}^{2}>11,070$

Uma possível explicação para a ausência de serviço de coleta de lixo é a localização geográfica dos bairros, pois os bairros que apresentaram o menor percentual de coleta são circunscritos da região rural da cidade de Coremas, a falta de políticas públicas e de investimento pode vir a acarretar sérios problemas ambientais causados pela disposição inadequado dos resíduos ou queima em alguns casos.

Vilar et al. (2010) afirma que o serviço de coleta de resíduos sólidos nos municípios deve ser regido por normas com o objetivo de delegar a responsabilidade de cada um (população, governo e entidade), onde a população deve ser sempre informada e orientada de como conduzir de forma adequada no sistema de coleta de resíduos sólidos, seja através de informativos ou de campanhas e palestras de educação. A ausência desse serviço também pode ser propagar em danos ao meio ambiente e a saúde pública, um vez que, há riscos de contaminação de rios e do próprio lençol freático, presença de animais (e de alguns tipos de vetores) que transmitirão doenças para os seres humanos e principalmente para os catadores de lixos.

Despertar o interesse da sociedade é de suma importância no intuito de promover uma mudança de hábitos e atitudes, pois é na origem, que se encontra um dos maiores problemas quanto á coleta de resíduos, sendo este muitas vezes disposto em locais inadequados em virtude de comodismo, trazendo diversos problemas sociais e ambientais.

Através do teste qui-quadrado identificou-se a relação de dependência entre as variáveis Se possui coleta de resíduos versus Destinação do lixo gerado em seu domicílio (Tabela 4). Assim nos bairros ou apenas em ruas que possuem 
a coleta de lixo é possível identificar uma participação significativa da população, onde grande parte tem a consciência dos impactos negativos do mesmo no meio e separa o lixo para a coleta pública. Embora exista a coleta pública em determinados bairros, ainda é possível identificar moradores que não colaboram com o sistema, seja lançando o lixo em terrenos baldios ou queimando o mesmo.

Tabela 4. Estatísticas do teste qui-quadrado para verificar relação entre as variáveis Se possui coleta de resíduos e Destinação do lixo gerado em seu domicílio.

\begin{tabular}{lr}
\hline Teste Qui-Quadrado & Valor \\
\hline Estatistica X & $54,88916128^{2}$ \\
Graus de Liberdade & 2 \\
P-Valor & $1,20495 \mathrm{E}-12$ \\
\hline *Rejeitar $\mathrm{H}_{0}$ (Hipótese nula) se $\mathrm{X}^{2}>5,991$ &
\end{tabular}

\section{CONCLUSÕES}

A escolaridade, a renda e o local de residência influenciam nas atitudes relacionadas ao destino dos resíduos sólidos dos residentes da Cidade de Coremas.

O comportamento ambiental e as atitudes dos moradores da cidade de Coremas é limitada, havendo uma grande necessidade de atenção do poder púbico para uma melhor gestão ambiental e subsidio de políticas que proporcionem alterações nos padrões de produção e consumo, no sentido de tornar o sistema mais sustentável.

\section{REFERÊNCIAS}

BIDONE, F. R. A.; POVINELLI, J. Conceitos básicos de resíduos sólidos. São Carlos: EESC/USP, 1999. 120p.

BRASIL. [Lei n. 12.305, de 2 de agosto de 2010]. Política nacional de resíduos sólidos [recurso eletrônico]. - 2. ed. Brasília : Câmara dos Deputados, Edições Câmara, 2012. 73p. (Série legislação; n.81), vista em http://fld.com.br/catadores/pdf/politica_residuos_solidos.pdf, visitado em 15 de julho de 2016.

BRASIL, Lei no 6.938, de 31 de Agosto de 1981. Dispõe sobre a Política Nacional do Meio Ambiente, seus fins e mecanismos de formulação e aplicação, e dá outras providências. 1981.

BRASIL, Lei $\mathrm{n}^{\circ} 11.445$, de 5 de janeiro de 2007. Diário Oficial da União. Brasília, 2007. Disponível em: < http://www.planalto.gov.br/ccivil_03/_ato2007-

2010/2007/lei/111445.htm>. Acesso em: 13 jun. 2016.

CANTER, L. W. Environmental impact assessment. New York: McGraw Hill Book, (Series in Water Resources and Environment Engineering) 1977. 331 p.

CARDOSO FILHO, G. T. A gestão de resíduos sólidos em Parintins/AM à luz da Política Nacional de Resíduos Sólidos. Revista Somanlu. Ano 12, nº 1, jan/jun 2012. Manaus, 2012.

CORNIERI, M. G.; FRACALANZA, A. P. Desafios do Lixo em nossa Sociedade. Revista Brasileira de Ciências Ambientais - Número 16 - Junho/2010.

FRANCO, T. R. Coleta seletiva de lixo domiciliar: estudos para implantação. Monografia (Bacharelado) - Instituto de Geografia, Universidade Federal de Uberlândia, Uberlândia, 2000 .

IBGE. INSTITUTO BRASILEIRO DE GEOGRAFIA E ESTATÍSTICA (BRASIL). Indicadores de desenvolvimento sustentável: Brasil 2015. Rio de Janeiro, RJ: IBGE, 2015. $352 \mathrm{p}$.

JACOBI, P. R. Educação ambiental: o desafio da construção de um pensamento crítico, complexo e reflexivo. Revista Educação e Pesquisa, São Paulo, v.31, n.2, p. 233-250, 2005.

LEFT, E. Saber ambiental: sustentabilidade, racionalidade, complexidade, poder. 35 a ed. Petrópolis: Vozes; 2001.

MINISTÉRIO DA INTEGRAÇÃO NACIONAL. Nova delimitação do semiárido brasileiro. Brasília: MIN/Secretaria de Políticas de Desenvolvimento Regional, 2005.

MUCELIN, C. A., BELLINI, M. Garbage and perceptible environmental impacts in urban ecosystem. Sociedade e Natureza. (Online), 2008, vol.20, no.1, ISSN 1982-4513. Similarity: 0.395305. SciELO Brasil.

VILAR, J. W. C.; CASADO, A. P. B.; BRASILEIRO, G. A. M.; BOMFIM, E. O.; SILVEIRA, A. M. ; Diagnóstico participativo da gestão dos resíduos sólidos do agreste central sergipano, 2010. In: $3^{\circ}$ Simpósio Ibero americano de Ingeniería de Resíduos e $2^{\circ}$ Seminário da Região Nordeste sobre Resíduos Sólidos, 2010, João Pessoa. Anais ... (Online), 2010 . 